\title{
Sphere to cylinder transition in a single phase microemulsion system: A theoretical investigation
}

Cite as: J. Chem. Phys. 115, 1073 (2001); https://doi.org/10.1063/1.1380428

Submitted: 30 October 2000 . Accepted: 30 April 2001 . Published Online: 02 July 2001

Edgar M. Blokhuis, and Wiebke F. C. Sager

\section{ARTICLES YOU MAY BE INTERESTED IN}

Structure and dynamics of water in nanoscopic spheres and tubes

The Journal of Chemical Physics 141, 18C535 (2014); https://doi.org/10.1063/1.4898380

Dynamics of bicontinuous microemulsion phases with and without amphiphilic blockcopolymers

The Journal of Chemical Physics 115, 9563 (2001); https://doi.org/10.1063/1.1413509

Self-assembly of surfactant liquid crystalline phases by Monte Carlo simulation

The Journal of Chemical Physics 91, 2479 (1989); https://doi.org/10.1063/1.457007

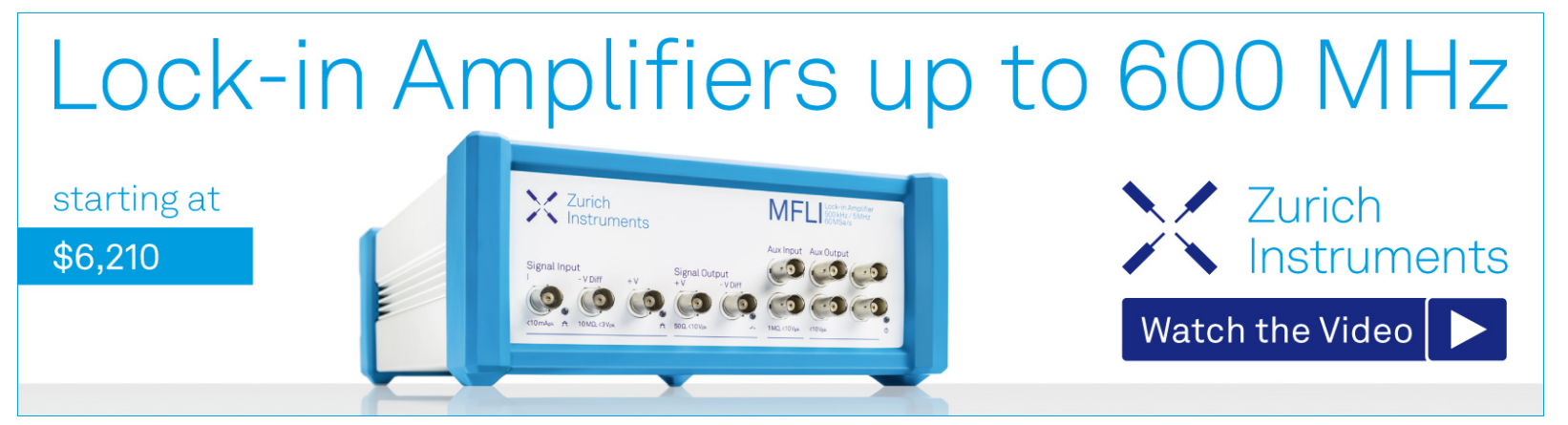

J. Chem. Phys. 115, 1073 (2001); https://doi.org/10.1063/1.1380428

115,1073

(C) 2001 American Institute of Physics. 


\title{
Sphere to cylinder transition in a single phase microemulsion system: A theoretical investigation
}

\author{
Edgar M. Blokhuis \\ Colloid and Interface Science, Leiden Institute of Chemistry, Gorlaeus Laboratories, P.O. Box 9502, \\ 2300 RA Leiden, The Netherlands \\ Wiebke F. C. Sager \\ KFA Jülich GmbH, Forschungszentrum, IFF, D-52425 Jülich, Germany
}

(Received 30 October 2000; accepted 30 April 2001)

\begin{abstract}
The sphere to cylinder transition in a one-phase droplet microemulsion system is studied theoretically. Within the framework of the curvature energy model by Helfrich, it was already shown by Safran et al. [J. Phys. (France) Lett. 45, L-69 (1984)] that for a certain range of the curvature parameters (rigidity constants and spontaneous curvature), a transition occurs from spherical droplets to infinitely long cylinders through a region where both spheres and cylinders are present. Our aim is to further investigate this region in a quantitative way by including - in addition to curvature energy - translation entropy, cylinder length polydispersity, and radial polydispersity. In this way we are able to obtain structural information on the spheres and cylinders formed, their respective volume fractions, and polydispersity, and provide a more detailed comparison with experimental results. (C) 2001 American Institute of Physics. [DOI: 10.1063/1.1380428]
\end{abstract}

\section{INTRODUCTION}

Over the past three decades it has been shown that microemulsions are structurally well-defined self-organizing mixtures of water, oil, and surfactants that can form a wide variety of thermodynamically stable phases. These comprise phases consisting of (more or less spherical) droplets of water in oil (w/o microemulsions or $\mathrm{L}_{2}$-phase) or oil droplets in water (o/w microemulsion or $\mathrm{L}_{1}$-phase), as well as bicontinuous mono- and bilayer phases. If the temperature and/or ionic strength of the aqueous phase is varied, a rich phase behavior is generally revealed, ${ }^{1,2}$ whereby microemulsion phases can coexist with water and/or oil excess phases as well as liquid crystalline phases forming two- and threephase equilibria.

Ample experimental evidence from, e.g., electric birefringence (Kerr-effect), dielectric spectroscopy, fluorescence quenching, turbidity, and temperature jump experiments, has been presented for droplet aggregation in the $\mathrm{L}_{1}$ and $\mathrm{L}_{2}$-phases. ${ }^{3}$ Furthermore, a considerable jump (2-3 orders of magnitude) in conductivity has been reported for w/o AOT microemulsions when the temperature or amount of internal phases is increased. ${ }^{4}$ It is still a matter of debate whether this increase in conductivity is a result of charge transfer between aggregated droplets or whether the aggregated droplets open up forming interconnected cylindrical structures. Even though the formation of cylindrical structures is extensively documented experimentally in micellar systems, ${ }^{5,6}$ their existence is less well-established in microemulsions.

Recent evidence for the formation of cylindrical structures has been supplied by SAXS and SANS scattering studies in the $\mathrm{L}_{1}$ and $\mathrm{L}_{2}$-phases. The usual interpretation of the scattering data in this region focuses on a fit of (aggregating) spherical droplets characterized by an average radius, radial polydispersity, and stickiness parameter describing the de- gree of aggregation. ${ }^{7,8}$ It was found by Ilgenfritz et al. ${ }^{9}$ and Glatter et al. ${ }^{10}$ that also cylindrical structures start to become present. Only recently could a more quantitative study be undertaken of this structural transition from spherical droplets to cylindrical structures in the one-phase microemulsion system. ${ }^{11}$ Using SAXS, the structural changes in w/o AOT microemulsions were investigated as a function of temperature $\left(15-60^{\circ} \mathrm{C}\right.$ ), salt concentration (up to $0.6 \% \mathrm{NaCl}$ ), water/AOT molar ratio (25-60), and droplet weight fraction $(2 \%-20 \%)^{11}$

Theoretically it now seems well-established that the Helfrich free energy ${ }^{12}$ describing the curvature free energy of the interfacial surfactant layer can help understand the global features of the microemulsion phase diagram. ${ }^{13,14}$ As in the experimental situation, while a lot is understood when it concerns the description of spherical microemulsion droplets, relatively little is known on the formation of cylindrical structures. Pivotal work has been done by Safran and co-workers. ${ }^{15-17}$ In 1984 the phase diagram of the sphere to cylinder transition was published based on Helfrich's curvature energy but neglecting entropy effects, ${ }^{14,15}$ while more recently entropy effects were included to predict closed-loop coexistence regions in a system consisting of spheres and cylinders. ${ }^{16}$ It is our intention in this article to extend the work by Safran and co-workers and to study the sphere to cylindrical transition in the one-phase region in more detail. Our aim is to investigate this in a quantitative way by including translation entropy, cylinder length polydispersity, and radial polydispersity.

Our theory has three important ingredients:

(1) Curvature free energy: The Helfrich form of the free energy is used to describe the interfacial free energy of the curved surfactant monolayer that separates the oil and the water phase, ${ }^{12}$ 


$$
F_{\text {curv }}=\int d A\left[-\frac{2 k}{R_{0}} J+\frac{k}{2} J^{2}+\bar{k} K\right] .
$$

The above free energy features an integral over the whole surface area, $A$, of the total curvature, $J=1 / R_{1}+1 / R_{2}$ and Gaussian curvature, $K=1 /\left(R_{1} R_{2}\right)$ with $R_{1}$ and $R_{2}$ the principal radii of curvature at a certain point on the surface $A$. Three curvature parameters are introduced: $1 / R_{0}$, the inverse radius of spontaneous curvature, $k$ the rigidity constant of bending, and $\bar{k}$ the rigidity constant associated with Gaussian curvature. In the following analysis, we treat these curvature parameters as unknown and construct our phase diagrams in terms of them. However, we do know from experiments that $k$ and $\bar{k}$ are approximately constant over the temperature range considered and of the order of 1 or a few $k_{B} T,{ }^{18}$ while the inverse radius of spontaneous curvature changes significantly as a function of temperature (approximately linear) and can even change sign at the so-called inversion temperature $\bar{T}$. ${ }^{19}$ Therefore, in the comparison with experiments we consider $k$ and $\bar{k}$ as constants and treat $1 / R_{0}$ as our "temperature variable," $1 / R_{0} \propto(T-\bar{T})$.

(2) Entropy: Although the consideration of the curvature free energy alone already gives good qualitative insight into the microemulsion phase diagram, entropy needs to be considered in any more quantitative analysis. Entropy is generally responsible for the occurence of polydispersity, which is an important feature of microemulsion systems, and it will smoothen structural transitions in the one-phase region like the sphere to cylindrical transition that we consider here. The theory for including entropy in microemulsion systems is however not free of controversy in the literature. ${ }^{20}$ In this article we investigate a number of different expressions for the entropic contribution to the free energy to find out which aspects are model dependent and which aspects are more generally valid.

(3) Constraints: Two constraints have to be considered. First, the total volume, $V_{\text {tot }}$, inside the spheres and cylinders is determined by the amount of internal phase present, for instance the amount of water when we consider water-in-oil microemulsions. Second, the total surface area, $A$, is determined by the amount of surfactant in the system. When we minimize the free energy consisting of the curvature free energy and the entropic contribution to the free energy, these two constraints have to be taken into account. One way to take these constraints into account is to add Lagrange multipliers (which we will call $\sigma$ and $-\Delta p$ ) to the free energy.

We start, in Sec. II, with a reinspection of the phase diagram of the sphere to cylindrical transition in which only the curvature free energy is taken into account with the above constraints neglecting the contribution of entropy. This phase diagram was first published by $\operatorname{Safran}^{14,15}$ and it already shows many features of the phase diagrams calculated in later sections when entropy is taken into account. In Sec. III, translation entropy is included and the cylinder length polydispersity is considered, while in Sec. IV also the polydispersity in the radius of the sphere and the cylinder is taken into account. In the final section we summarize our findings and discuss the limitations of the theory presented.

\section{NO ENTROPY: SPHERES AND INFINITELY LONG CYLINDERS}

The curvature free energy of $N_{s}$ spheres with radius $R_{s}$ is derived by inserting $J=2 / R_{s}$ and $K=1 / R_{s}^{2}$ into Eq. (1.1),

$$
\frac{F_{\text {curv }, s}}{\pi k}=N_{s}\left\{-\frac{16}{R_{0}} R_{s}+4(2+x)\right\},
$$

with $x \equiv \bar{k} / k$ defined as the ratio of the two rigidity constants. The total volume and surface area are given by

$$
\begin{aligned}
& A=N_{s} 4 \pi R_{s}^{2}, \\
& V_{\text {tot }}=N_{s} \frac{4}{3} \pi R_{s}^{3} .
\end{aligned}
$$

The curvature free energy of $N_{c}$ cylinders with radius $R_{c}$ and length $L \gg R_{c}$ (so that we can neglect the curvature energy of the ends of the cylinder) is derived by inserting $J=1 / R_{c}$ and $K=0$ into Eq. (1.1),

$$
\frac{F_{\text {curv }, c}}{\pi k}=N_{c} L\left\{-\frac{4}{R_{0}}+\frac{1}{R_{c}}\right\} .
$$

The volume and surface area are given by

$$
\begin{aligned}
& A=N_{c} 2 \pi R_{c} L, \\
& V_{\text {tot }}=N_{c} \pi R_{c}^{2} L .
\end{aligned}
$$

The free energy of a system containing both spheres and cylinders is the sum of the above free energies of $N_{s}$ spheres and $N_{c}$ cylinders. Instead of $N_{s}$ and $N_{c}$ as parameters, it is more convenient to use the volume fractions of spheres and cylinders, $v_{s}$ and $v_{c}$, defined as

$$
\begin{aligned}
& v_{s} \equiv \frac{N_{s}}{V_{\mathrm{tot}}} \frac{4}{3} \pi R_{s}^{3}, \\
& v_{c} \equiv \frac{N_{c}}{V_{\mathrm{tot}}} \pi R_{c}^{2} L .
\end{aligned}
$$

The total free energy then becomes

$$
\begin{aligned}
\frac{F_{\text {curv }}}{k V_{\text {tot }}}= & v_{s}\left\{-\frac{12}{R_{0}} \frac{1}{R_{s}^{2}}+3(2+x) \frac{1}{R_{s}^{3}}\right\} \\
& +v_{c}\left\{-\frac{4}{R_{0}} \frac{1}{R_{c}^{2}}+\frac{1}{R_{c}^{3}}\right\},
\end{aligned}
$$

with the volume and area constraints written as

$$
\begin{aligned}
& v_{s}+v_{c}=1, \\
& \frac{3 v_{s}}{R_{s}}+\frac{2 v_{c}}{R_{c}}=\frac{A}{V_{\text {tot }}} \equiv \frac{1}{\omega} .
\end{aligned}
$$

The ratio between the total volume and surface area defines the length scale $\omega$. In general, $\omega$ depends on the size of the surfactant molecules and the molecules constituting the internal phase. Specifically, $A=n_{\text {surf }} a_{\text {surf }}$, with $n_{\text {surf }}$ the number of surfactant molecules and $a_{\text {surf }}$ the surface area taken in by a surfactant molecule; $V_{\text {tot }}=n_{\text {int }} v_{\text {int }}$, with $n_{\text {int }}$ the number of molecules in the internal phase and $v_{\text {int }}$ the volume per molecule taken in by the internal phase.

In the following we express all lengths $\left(R_{s}, R_{c}, R_{0}\right.$, and $L$ ) in terms of $\omega$. For instance, in the case that the minimi- 
zation of the total curvature free energy yields $v_{s}=1$ (only spheres), one immediately finds from the second constraint in Eq. (2.7) that $R_{s}=3$, in units of $\omega$. Analogously, when only cylinders are present $\left(v_{c}=1\right)$, the radius of the cylinder is given by $R_{c}=2$, in units of $\omega$. In these cases, with only one species present, the radii therefore follow directly from the constraints. The free energies are then simply obtained from Eq. (2.6),

$$
\begin{array}{ll}
\frac{\omega^{3} F_{\text {curv }}}{k V_{\text {tot }}}=-\frac{4}{3} \frac{1}{R_{0}}+\frac{1}{9}(2+x), & \text { only } \text { spheres } \\
\frac{\omega^{3} F_{\text {curv }}}{k V_{\text {tot }}}=-\frac{1}{R_{0}}+\frac{1}{8}, & \text { only cylinders. }
\end{array}
$$

When we consider the free energy of the system consisting of both spheres and cylinders, the radii have to be determined from the minimization of the free energy in Eq. (2.6) with the constraints in Eq. (2.7). A convenient way is to first solve the volume fractions $v_{s}$ and $v_{c}$ in terms of $R_{c}$ and $R_{s}$ from Eq. (2.7), yielding

$$
\begin{aligned}
& v_{s}=\frac{R_{s}\left(2-R_{c}\right)}{2 R_{s}-3 R_{c}}, \\
& v_{c}=\frac{R_{c}\left(R_{s}-3\right)}{2 R_{s}-3 R_{c}},
\end{aligned}
$$

and insert the result into the free energy in Eq. (2.6). The minimizing equations $\partial F / \partial R_{s}=0$ and $\partial F / \partial R_{c}=0$ then yield the following pair of algebraic equations to determine $R_{S}$ and $R_{c}$ in terms of $x$ and $1 / R_{0}$ :

$$
\begin{aligned}
& \frac{4}{R_{0}} R_{s} R_{c}\left(R_{s}^{2}+3 R_{c}^{2}-4 R_{s} R_{c}\right) \\
& \quad+6(2+x) R_{c}^{2}\left(R_{s}-R_{c}\right)-R_{s}^{3}=0, \\
& -\frac{8}{R_{0}} R_{s} R_{c}\left(R_{s}^{2}+3 R_{c}^{2}-3 R_{s} R_{c}\right) \\
& \quad+6(2+x) R_{c}^{3}+R_{s}^{2}\left(4 R_{s}-9 R_{c}\right)=0 .
\end{aligned}
$$

With the free energies of the three systems (spheres, cylinders, and spheres+cylinders) determined, one is then able to construct the phase boundaries of the transitions between spheres and cylinders and between these two phases and the phase consisting of both spheres and cylinders. It should be realized that the phase consisting of spheres and cylinders is still a single phase and not phase separated.

Before showing the complete phase diagram we first need to discuss the transition to two other phases. It turns out that for small values of $1 / R_{0}$ (large $R_{0}$ ) a transition occurs to the lamellar phase, while for large values of $1 / R_{0}\left(\operatorname{small} R_{0}\right)$ the internal phase is expelled as an excess phase and phase separation occurs. The boundary at which the latter transition occurs is termed the solubilization limit or the emulsification failure transition. These two phases are now discussed in more detail.

\section{A. Solubilization limit}

At the solubilization limit (SL), the internal phase starts to be present as an excess phase. If the volume of the excess phase is denoted by $V_{0}$ and $v_{0}$ is defined as $v_{0} \equiv V_{0} / V_{\text {tot }}$, the volume constraint in Eq. (2.7) becomes

$$
v_{0}+v_{s}+v_{c}=1 .
$$

The amount of internal phase that is expelled as an excess phase, $v_{0}$, is determined by a minimization of the free energy with respect to $v_{0}$. The solubilization limit is therefore determined by the minimization equation $\partial F / \partial v_{0}=0$, with the condition that $v_{0}=0$ at the solubilization limit. This procedure gives as solubilization limit for the three systems, spheres, cylinders, and spheres + cylinders, the following relations between $1 / R_{0}$ and $x$ :

$$
\begin{array}{ll}
\frac{1}{R_{0}}=\frac{1}{6}(2+x), & \text { SL, only spheres, } \\
x=0, & \text { SL, spheres + cylinders, } \\
\frac{1}{R_{0}}=\frac{1}{4}, & \text { SL, only cylinders. }
\end{array}
$$

\section{B. Transition to the lamellar phase}

The lamellar phase $\left(L_{\alpha}\right)$ is characterized by planar sheets of surfactant films that carry no curvature so that the corresponding curvature energy is zero $\left(F_{\text {curv }}=0\right)$. Therefore, when the calculated curvature energy of the spheres and cylinders changes sign and becomes positive, the free energy for forming spheres and/or cylinders is higher than the free energy associated with the lamellar phase and a (first order) phase transition occurs. The location of the transition to the lamellar phase is thus determined by inserting $F_{\text {curv }}=0$ into Eq. (2.6). One finds

$$
\begin{aligned}
& \frac{1}{R_{0}}=\frac{1}{12}(2+x), \quad L_{\alpha}, \text { only spheres, } \\
& \frac{1}{R_{0}}=\frac{1}{8}, \quad L_{\alpha} \text {, only cylinders. }
\end{aligned}
$$

The expression for $1 / R_{0}$ at the transition to the lamellar phase of the phase comprising spheres and cylinders is somewhat tedious and we will not reproduce it here. It can be derived from solving the set of equations in Eq. (2.10) together with the condition,

$$
\begin{aligned}
& \frac{4}{R_{0}} R_{s} R_{c}\left(R_{s}^{2}-3 R_{s}+6 R_{c}-3 R_{c}^{2}\right)+3(2+x) R_{c}^{2}\left(R_{c}-2\right) \\
& \quad+R_{s}^{2}\left(3-R_{s}\right)=0,
\end{aligned}
$$

for $R_{s}, R_{c}$, and $1 / R_{0}$.

The resulting phase diagram as a function of $\omega / R_{0}$ and $x \equiv \bar{k} / k$ is shown in Fig. 1. With slightly different axes, it was already published by Safran. ${ }^{14,15}$ The upper region is the $\overline{2} \varphi$-region where the microemulsion coexists with the internal phase, the lower region is the lamellar phase $\left(L_{\alpha}\right)$, and in between is the one-phase microemulsion region, which consists of either spheres, or cylinders, or spheres and cylinders 


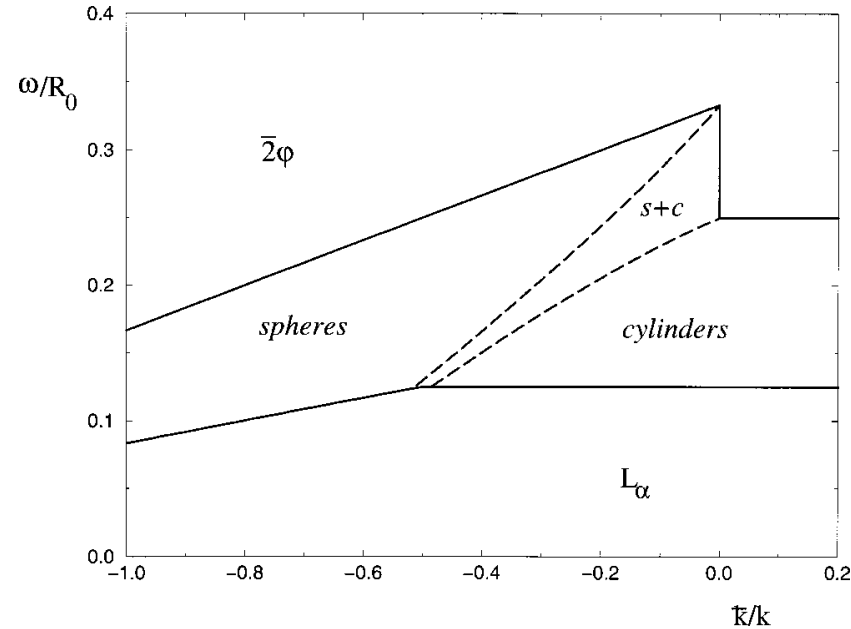

FIG. 1. Microemulsion phase diagram without entropy as a function of $\omega / R_{0}$ and $\bar{k} / k$. The upper region is the $\overline{2} \varphi$-region where the microemulsion coexists with the internal phase. The lower region is the lamellar phase $\left(L_{\alpha}\right)$. In between is the one-phase microemulsion region, which consists of either spheres, or (infinitely long) cylinders, or spheres and cylinders $(\mathrm{s}+\mathrm{c})$ with sharp transitions (dashed lines) between them.

$(\mathrm{s}+\mathrm{c})$ with sharp transitions (dashed lines) between them. It should be emphasized that in the calculation of the phase diagram in Fig. 1, we have only considered spheres and (infinitely long) cylinders as structures possibly present in the microemulsion phase. More complex structures, such as saddlelike structures, are therefore not considered but are expected to play a role when $x>0$.

\section{TRANSLATIONAL ENTROPY: SPHERES AND SPHEROCYLINDERS}

Next, we consider the influence of translational entropy on the phase diagram. For the cylinders, our treatment of the influence of entropy is very much in the sprit of the FloryHuggins theory of polymers. ${ }^{22}$ We first consider the entropic contribution to the free energy of spheres only.

\section{A. Spheres}

Subdividing the volume $V$ into volume elements $v_{0, s}$ each containing one or none spherical droplets, the entropy contribution of $N_{s}$ spheres is given by

$$
F_{\text {ent }, s}=k_{B} T\left[N_{s} \ln \left(\frac{N_{s} v_{0, s}}{V}\right)-N_{s}\right],
$$

where $k_{B}$ is Boltzmann's constant and $T$ the absolute temperature. In writing Eq. (3.1) we have assumed that the total volume occupied by the spherical particles is much smaller than the volume of the vessel, $V_{\text {tot }} \ll V$. The total free energy is the sum of the curvature energy and translational entropy

$$
\frac{F_{s}}{\pi k}=N_{s}\left\{-\frac{16}{R_{0}} R_{s}+4(2+x)+t\left[\ln \left(\frac{N_{s} v_{0, s}}{V}\right)-1\right]\right\} \text {, }
$$

where we have defined the reduced temperature, or reduced inverse rigidity constant of bending for that matter, $t$ $\equiv k_{B} T /(\pi k)$. The volume and area constraints are still given by Eq. (2.2),

$$
\begin{aligned}
& A=N_{s} 4 \pi R_{s}^{2}, \\
& V_{\text {tot }}=N_{s} \frac{4}{3} \pi R_{s}^{3} .
\end{aligned}
$$

The unknown volume element $v_{0, s}$ plays the role of the de Broglie volume $\Lambda^{3}$. The cubic root of $v_{0, s}$ is the typical length scale over which a microemulsion droplet needs to be displaced in order for it to "count" as constituting a different state. Its magnitude and scaling with, e.g., the droplet size is a matter of some debate. ${ }^{20}$ Within the context of statistical mechanical treatments using the curvature energy model, ${ }^{23}$ it now seems well-established that the hypothesis of Safran and co-workers ${ }^{14,16}$ to assume $v_{0, s}^{1 / 3}$ to be of the order of the droplet radius itself is a "reasonable approximation." ${ }^{20}$ In the present treatment we therefore take $v_{0, s}$ to be of the order of the droplet size,

$$
v_{0, s} \approx \frac{4 \pi}{3} R_{s}^{3} \text {. }
$$

Introducing the total volume fraction $\phi \equiv V_{\text {tot }} / V$, the free energy can then be written as

$$
\frac{F_{s}}{\pi k}=N_{s}\left\{-\frac{16}{R_{0}} R_{s}+4(2+x)+t[\ln (\phi)-1]\right\} .
$$

As in Sec. II, when only spheres are present, the spherical droplet radius follows directly from the constraints: $R_{s}=3$ (in units of $\omega$ ). The free energy $F_{s}$ is then simply obtained by substituting $R_{S}=3$ into the expression above. Different expressions for the entropic contribution can and have been proposed based either on a different assumption for the form of $v_{0, s}$ or taking droplet-droplet interactions into account. ${ }^{21}$ Typically, these alternate expressions lead to a slightly modified phase diagram not affecting the overall character of it.

\section{Solubilization limit}

The solubilization limit is derived in the same way as in the previous section. For the spheres alone one finds

$$
\frac{1}{R_{0}}=\frac{1}{6}(2+x)+\frac{t}{48}[2 \ln (\phi)-3] .
$$

In the literature it is more common to assume that $v_{0, s}$ is constant and the above expression for the solubilization limit becomes $^{23}$

$$
\frac{1}{R_{0}}=\frac{1}{6}(2+x)+\frac{t}{24} \ln \left(\frac{\phi v_{0, s}}{\frac{4}{3} \pi R_{s}^{3}}\right),
$$

with $R_{s}=3$.

\section{Transition to the lamellar phase}

The transition to the lamellar phase is simply determined by setting $F_{s}=0$ in Eq. (3.5) giving

$$
\frac{1}{R_{0}}=\frac{1}{12}(2+x)+\frac{t}{48}[\ln (\phi)-1] \text {. }
$$

\section{B. Spherocylinders}

In order to derive an expression for the entropy of the cylinder we need to take the length of the cylinder into con- 
sideration. In the Flory-Huggins theory for polymers, ${ }^{22}$ the length is expressed as $L=n l_{0}$ with $n$ the number of monomers with length $l_{0}$. The entropic contribution to the free energy of $N_{c}$ cylinders, all having length $L$, on a lattice with volume element $v_{0, c}$ is, in the Flory-Huggins mean-field approximation, given by

$$
F_{\text {ent }, c}=k_{B} T\left[N_{c} \ln \left(\frac{N_{c} v_{0, c}}{V}\right)-N_{c} n\right] .
$$

When we consider the curvature energy of cylinders of finite length $L$, the curvature energy associated with the "endcaps" of the cylinder needs to be considered. A full treatment to determine the end-cap energy involves the minimization of the free energy with respect to the full shape. ${ }^{17}$ In the present treatment, however, we assume the shape to be that of a "sphero-cylinder" (spherical end-caps) and only minimize with respect to the two shape parameters defining the spherocylinder: the radius $R_{c}$ and length $L$. The total free energy of spherocylinders with entropy is then

$$
\begin{aligned}
\frac{F_{c}}{\pi k}= & N_{c}\left\{-\frac{4 L}{R_{0}}+\frac{L}{R_{c}}-\frac{16}{R_{0}} R_{c}+4(2+x)\right. \\
& \left.+t\left[\ln \left(\frac{N_{c} v_{0, c}}{V}\right)-\frac{L}{l_{0}}\right]\right\},
\end{aligned}
$$

with the volume and surface area given by

$$
\begin{aligned}
& A=N_{c} 2 \pi R_{c}\left(L+2 R_{c}\right), \\
& V_{\text {tot }}=N_{c} \frac{\pi}{3} R_{c}^{2}\left(3 L+4 R_{c}\right) .
\end{aligned}
$$

In reality, the cylinders are not all of the same length $L$ and one should consider the effect of polydispersity in the cylinder length.

\section{Length polydispersity}

In order to account for polydispersity in the cylinder length, we need to allow for a distribution $N_{c}(n)$ denoting the number of cylinders with length $L=n l_{0}$. The total free energy then becomes a functional of the distribution $N_{c}(n)$,

$$
\begin{aligned}
\frac{F_{c}}{\pi k}= & \sum_{n} N_{c}(n)\left\{-\frac{4 n l_{0}}{R_{0}}+\frac{n l_{0}}{R_{c}}-\frac{16}{R_{0}} R_{c}+4(2+x)\right. \\
& \left.+t\left[\ln \left(\frac{N_{c}(n) v_{0, c}}{V}\right)-n\right]\right\},
\end{aligned}
$$

with the volume and surface area given by

$$
\begin{aligned}
& A=\sum_{n} N_{c}(n) 2 \pi R_{c}\left(n l_{0}+2 R_{c}\right), \\
& V_{\text {tot }}=\sum_{n} N_{c}(n) \frac{\pi}{3} R_{c}^{2}\left(3 n l_{0}+4 R_{c}\right) .
\end{aligned}
$$

In the following we replace the summation by an integration over $n$. The minimization of the free energy in Eq. (3.12), taking the above constraints on the volume and surface area into account, is done in two steps. First, it is noted that the functional differentiation with respect to $N_{c}(n)$ yields an exponential distribution for $N_{c}(n)$,

$$
N_{c}(n)=\frac{V}{v_{0, c}} e^{-\alpha n+\beta}
$$

with $\alpha$ and $\beta$ constants to be determined from a further minimization. Depending on the molecular model used, the volume $v_{0, c}$ might depend on $n$, so that the exponential distribution above may have an algebraic prefactor. In the present treatment we take $v_{0, c}$ independent of $n$.

Instead of $\alpha$ and $\beta$ as parameters, it is more convenient ${ }^{24}$ to express the exponential distribution in terms of the average length, $L$, and average number of cylinders, $N_{c}$, defined as

$$
\begin{aligned}
& N_{c} \equiv \int_{0}^{\infty} \operatorname{dn} N_{c}(n), \\
& L \equiv \frac{1}{N_{c}} \int_{0}^{\infty} d n N_{c}(n) n l_{0},
\end{aligned}
$$

so that the distribution [Eq. (3.14)] becomes

$$
N_{c}(n)=\frac{N_{c} l_{0}}{L} e^{-\left(n l_{0} / L\right)} .
$$

As a second step, we insert the above exponential distribution back into the expression for the free energy in Eq. (3.12) and carry out the integration over $n .{ }^{24}$ We find an expression for the free energy quite similar to the expression for the free energy without length polydispersity [Eq. (3.10)],

$$
\begin{aligned}
\frac{F_{c}}{\pi k}= & N_{c}\left\{-\frac{4 L}{R_{0}}+\frac{L}{R_{c}}-\frac{16}{R_{0}} R_{c}+4(2+x)\right. \\
& \left.+t\left[\ln \left(\frac{N_{c} v_{0, c} l_{0}}{V L}\right)-\frac{L}{l_{0}}-1\right]\right\},
\end{aligned}
$$

with the boundary conditions of the same form as in Eq. (3.11),

$$
\begin{aligned}
& A=N_{c} 2 \pi R_{c}\left(L+2 R_{c}\right), \\
& V_{\text {tot }}=N_{c} \frac{\pi}{3} R_{c}^{2}\left(3 L+4 R_{c}\right) .
\end{aligned}
$$

Again, certain assumptions need to be made regarding the unknown volume element $v_{0, c}$ and the length scale $l_{0}$, which is the length scale over which two cylinders need to differ in length in order for the two cylinders to "count" as having different lengths. Similar to the case of spherical droplets, we assume that $v_{0, c}$ is of the order of a cylindrical segment with radius $R_{c}$ and length $l_{0}$,

$$
v_{0, c} \approx \pi R_{c}^{2} l_{0},
$$

and assume $l_{0}$ to be of the order of the radius of the cylinder,

$$
l_{0} \approx R_{c} \text {. }
$$

Other approximations are certainly possible and one could argue that $v_{0, c}^{1 / 3}$ and $l_{0}$ are fixed microscopic length scales to be determined by some other method. The assumptions in Eqs. (3.19) and (3.20) have the advantage that no additional unknown parameters have to be introduced. In the context of the approximations made, this certainly suffices but below other approaches will be considered. 
With the total volume fraction $\phi \equiv V_{\text {tot }} / V$, the free energy in Eq. (3.17) is written as

$$
\begin{aligned}
\frac{F_{c}}{\pi k}= & N_{c}\left\{-\frac{4 L}{R_{0}}+\frac{L}{R_{c}}-\frac{16}{R_{0}} R_{c}+4(2+x)\right. \\
& \left.+t\left[\ln \left(\frac{\phi R_{c}^{2}}{L^{2}}\right)-\frac{L}{R_{c}}-1\right]\right\},
\end{aligned}
$$

where we have neglected terms of $\mathcal{O}(1 / L)$.

In the case that only cylinders are present, the above free energy needs to be minimized with respect to $R_{c}, L$, and $N_{c}$ keeping the volume and surface area constraints in mind. One finds that $R_{c}$ and $L$ are determined by the following two equations:

$$
\begin{aligned}
& -\frac{8 R_{c}}{R_{0}}+(7+6 x)-\frac{4 R_{c}}{L}+\frac{3}{2} t\left[\ln \left(\frac{\phi R_{c}^{2}}{L^{2}}\right)+1\right] \\
& +5 t\left(1+\frac{14}{5} \frac{R_{c}}{L}\right)=0
\end{aligned}
$$

$\frac{6}{R_{c}} \frac{\left(L+2 R_{c}\right)}{\left(3 L+4 R_{c}\right)}=1$.

To leading order in $1 / L$ these two equations are solved explicitly to yield

$$
\begin{aligned}
R_{c}=2+ & \frac{8}{3} \frac{1}{L}+\mathcal{O}\left(\frac{1}{L^{2}}\right) \\
t \ln (L)= & -\frac{16}{3} \frac{1}{R_{0}}+\frac{1}{3}(7+6 x)+\frac{t}{2}\left[\ln (4 \phi)+\frac{13}{3}\right] \\
& +\mathcal{O}\left(\frac{1}{L}\right) .
\end{aligned}
$$

Furthermore, the solubilization limit and transition to the lamellar phase can be determined.

\section{Solubilization limit}

The solubilization limit is given by

$$
\frac{1}{R_{0}}=\frac{1}{4}(1-t)+\frac{1}{L}\left(\frac{2}{3}-\frac{23}{12} t\right)+\mathcal{O}\left(\frac{1}{L^{2}}\right),
$$

with the cylinder length $L$ at the solubilization limit to leading order given by

$$
t \ln (L)=(1+2 x)+\frac{t}{2}[\ln (4 \phi)+7]+\mathcal{O}\left(\frac{1}{L}\right),
$$

which is derived by inserting the expression for $1 / R_{0}$ in Eq. (3.25) into Eq. (3.24).

\section{Transition to the lamellar phase}

The transition to the lamellar phase is determined by setting $F_{c}=0$ in Eq. (3.21)

$$
\frac{1}{R_{0}}=\frac{1}{8}(1-t)-\frac{t}{2 L}+\mathcal{O}\left(\frac{1}{L^{2}}\right),
$$

with to leading order in $L$,

$$
t \ln (L)=\frac{1}{3}(5+6 x)+\frac{t}{2}\left[\ln (4 \phi)+\frac{17}{3}\right]+\mathcal{O}\left(\frac{1}{L}\right),
$$

which is derived by inserting the expression for $1 / R_{0}$ in Eq. (3.27) into Eq. (3.24).

In the calculation of the two conditions in Eqs. (3.22) and (3.23), we have made use of the expressions for $v_{0, c}$ and $l_{0}$ in Eqs. (3.19) and (3.20). As an aside we investigate the consequences of assuming that $v_{0, c}$ and $l_{0}$ are constants instead. One finds that the condition in Eq. (3.22) now becomes

$$
\begin{aligned}
& -\frac{8 R_{c}}{R_{0}}+(7+6 x)-\frac{4 R_{c}}{L}+\frac{3}{2} t\left[\ln \left(\frac{\phi v_{0, c} l_{0}}{\pi R_{c}^{2} L^{2}}\right)+1\right] \\
& +4 t \frac{R_{c}}{l_{0}}\left(1+\frac{R_{c}}{L}+\frac{l_{0}}{L}\right)=0 .
\end{aligned}
$$

The difference in approach only shows up as an endcorrection to the last term in Eq. (3.22). As argued before, such detail is lost in the approximative scheme considered here.

Having derived the free energies of spheres and cylinders separately, it is now easy to construct the free energy of the system containing both spheres and cylinders.

\section{Spheres and spherocylinders}

The total free energy of spheres and spherocylinders with length polydispersity is the sum of the free energies in Eqs. (3.5) and (3.21) taking the respective volume fractions into account,

$$
\begin{aligned}
\frac{\omega^{3} F}{k V_{\mathrm{tot}}}= & v_{s}\left\{-\frac{12}{R_{0}} \frac{1}{R_{s}^{2}}+3(2+x) \frac{1}{R_{s}^{3}}+t \frac{3}{4 R_{s}^{3}}\left[\ln \left(\phi v_{s}\right)-1\right]\right\} \\
& +\frac{3 v_{c} L}{\left(3 L+4 R_{c}\right)}\left\{-\frac{4}{R_{0}} \frac{1}{R_{c}^{2}}+\frac{1}{R_{c}^{3}}-\frac{16}{R_{0}} \frac{1}{R_{c} L}\right. \\
& \left.+4(2+x) \frac{1}{R_{c}^{2} L}+t \frac{1}{R_{c}^{2} L}\left[\ln \left(\frac{\phi v_{c} R_{c}^{2}}{L^{2}}\right)-\frac{L}{R_{c}}-1\right]\right\},
\end{aligned}
$$

with the volume and area constraints given by

$$
\begin{aligned}
& v_{s}+v_{c}=1, \\
& \frac{3 v_{s}}{R_{s}}+6 \frac{v_{c}}{R_{c}} \frac{\left(L+2 R_{c}\right)}{\left(3 L+4 R_{c}\right)}=1 .
\end{aligned}
$$

The free energy above needs to be minimized with respect to the five variables $R_{s}, R_{c}, L, v_{c}$, and $v_{s}$ with the two constraints in Eq. (3.31). This has been done, numerically, with the result shown in Fig. 2. In this example we have fixed the 

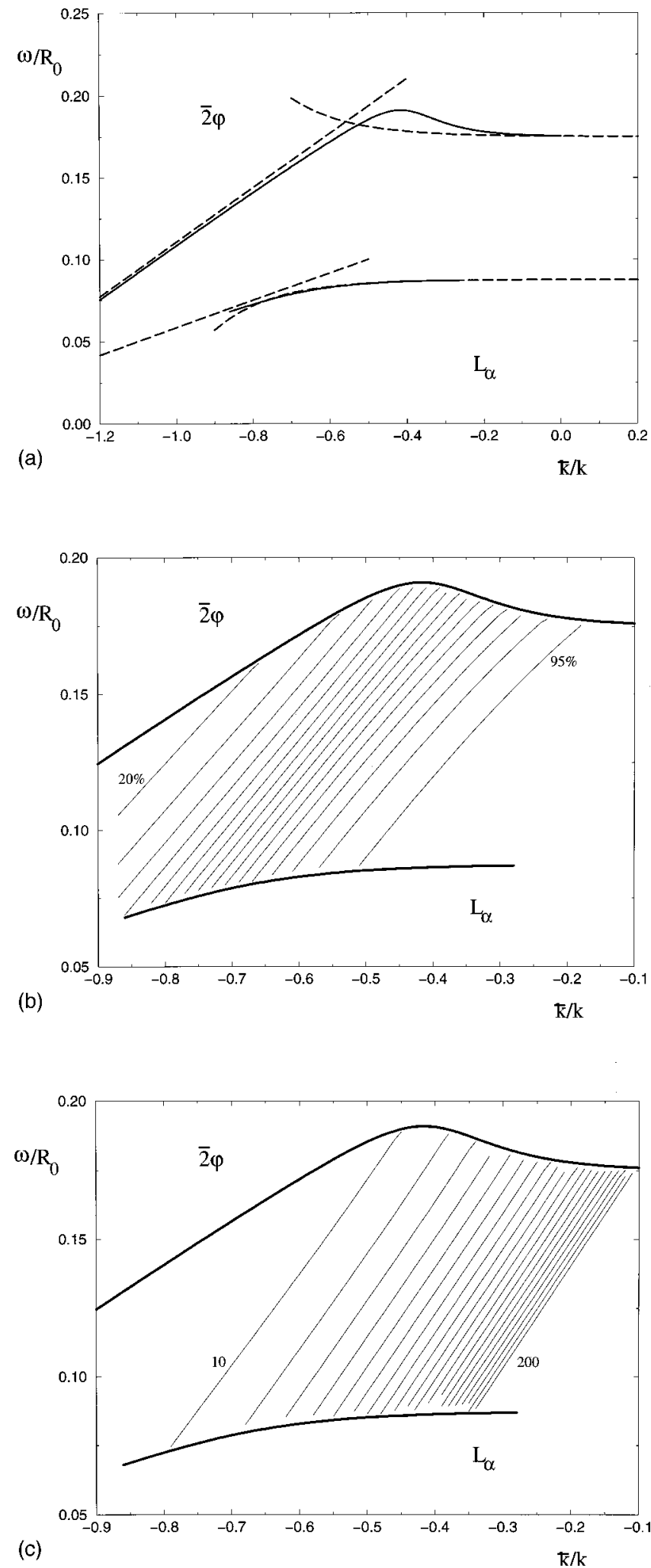

FIG. 2. Microemulsion phase diagram with translational entropy and cylinder length polydispersity, as a function of $\omega / R_{0}$ and $\bar{k} / k$ with $\phi=0.05$ and $t=0.3$. The drawn lines denote the location of the solubilization limit and the transition to the lamellar phase. In (a) the dashed lines are the limiting analytical results for the solubilization limit and transition to the lamellar phases as given in Eqs. (3.6), (3.8), (3.25), and (3.27). The cylinder volume fraction, $v_{c}=20 \%-95 \%$ (steps of $5 \%$ ), and average cylinder length, $L=10-200$ (steps of 10, in units of $\omega$ ) are shown in (b) and (c), respectively. droplet volume fraction $\phi=0.05$ and set the reduced temperature $t=0.3$ (which corresponds to $k \approx 1 \quad k_{B} T$ ). The dashed lines in Fig. 2(a) are the limiting analytical results for the solubilization limit and transition to the lamellar phases as given in Eqs. (3.6), (3.8), (3.25), and (3.27). An important distinction with the phase diagram in Fig. 1 is the fact that there is no sharp transition to a region with only spheres or only cylinders. The inclusion of translational entropy therefore smoothens the sphere to cylinder transition. This means that at any finite temperature the relative population of spheres and cylinders is determined by the Boltzmann distribution prohibiting the existence of regions with only spheres or cylinders present.

In Figs. 2(b) and 2(c), the cylinder volume fraction and cylinder length, respectively, are shown in the phase diagram. In the direction of increasing $x \equiv \bar{k} / k$ and decreasing $\omega / R_{0}$ both the cylinder volume fraction and cylinder length increases.

Already the (numerical) minimization of the free energy in Eq. (3.30) gives a good indication of the influence of entropy on the phase diagram of spherical and cylindrical microemulsions. For the comparison with the experimental phase diagram, however, we still need to consider one additional effect: the polydispersity in the radius of the spheres and cylinders.

\section{RADIAL POLYDISPERSITY}

In this section we account for the polydispersity in the radius of the spherical and cylindrical structures. As in the case of length polydispersity, we now have a distribution $N_{s}(n)\left(N_{c}(n)\right)$ denoting the number of spheres (cylinders) with radius $R_{s}=n r_{0, s}\left(R_{c}=n r_{0, c}\right)$.

\section{A. Spheres}

We first consider the free energy of spherical droplets,

$$
\begin{aligned}
\frac{F_{s}}{\pi k}= & \sum_{n} N_{s}(n)\left\{-\frac{16}{R_{0}} n r_{0, s}+4(2+x)\right. \\
& \left.+t\left[\ln \left(\frac{N_{s}(n) v_{0, s}}{V}\right)-1\right]\right\},
\end{aligned}
$$

with the volume and surface area now given by

$$
\begin{aligned}
& A=\sum_{n} N_{s}(n) 4 \pi\left(n r_{0, s}\right)^{2}, \\
& V_{\mathrm{tot}}=\sum_{n} N_{s}(n) \frac{4 \pi}{3}\left(n r_{0, s}\right)^{3} .
\end{aligned}
$$

Again, the summation is replaced by an integration over $n$. The above free energy is minimized adding Lagrange multipliers $\sigma$ and $-\Delta p$ fixing the surface area and volume, respectively. The distribution then has the form, 


$$
N_{s}(n)=\frac{V}{v_{0, s}} e^{-(1 / t)\left[4(2+x)-\left(16 / R_{0}\right) n r_{0, s}+(\sigma / k) 4\left(n r_{0, s}\right)^{2}-(\Delta p / k)(4 / 3)\left(n r_{0, s}\right)^{3}\right]} .
$$

Again, the above distribution may have an algebraic prefactor due to some assumed $n$ (radial) dependence of $v_{0, s}$. The above distribution was first derived by Overbeek. ${ }^{25}$ Following Reiss, ${ }^{26} v_{0, s}$ was taken proportional to $n^{-3 / 2}$ by Overbeek but different models leading to different exponents of the prefactor have been reported in the literature. ${ }^{27,28}$ Here we proceed by assuming that $r_{0, s}$ is some microscopic length scale to be determined in some other way while the expression for $v_{0, s}$ is the same as in the previous section [cf. Eq. (3.4)],

$$
v_{0, s} \approx \frac{4 \pi}{3} R_{s}^{3}
$$

We should now proceed in a similar way as in the treatment of the length polydispersity of the cylinder: (1) assume some form for $v_{0, s}$ such as in Eq. (4.4), (2) express the Lagrange multipliers in terms of an average radius and total number of droplets, (3) insert the resulting distribution into the free energy, and (4) minimize with respect to the remaining variables. This route is, however, mathematically rather complicated if no further approximations are made. ${ }^{29}$ What we will do here is to approximate the distribution in Eq. (4.3) by discarding the terms proportional to $n^{2}$ and $n^{3}$ in the exponent and allow for the presence of some algebraic prefactor with an exponent which we will call $z_{s}$. With this approximation, the important characteristics of the distribution remain with the neglect of the $n^{2}$ and $n^{3}$-terms in the exponent only affecting the tail-end of the distribution. The advantage of this approach is that the resulting distribution has the form of the well-known Schultz distribution, ${ }^{30}$ widely used in the experimental $\mathrm{fit}^{7,11}$ of the size-distribtion of microemulsion droplets,

$$
N_{s}(n)=\frac{N_{s} r_{0, s}}{R_{s}} \frac{\left(z_{s}+1\right)^{z_{s}+1}}{\Gamma\left(z_{s}+1\right)}\left(\frac{n r_{0, s}}{R_{s}}\right)^{z_{s}} e^{-\left(z_{s}+1\right) n r_{0, s} / R_{s}},
$$

where $\Gamma(x)$ is Euler's Gamma function, and where $N_{s}$ and $R_{s}$ are the total number of droplets and average radius defined by

$$
\begin{aligned}
& N_{s} \equiv \int_{0}^{\infty} d n N_{s}(n), \\
& R_{s} \equiv \frac{1}{N_{s}} \int_{0}^{\infty} d n N_{s}(n) n r_{0, s} .
\end{aligned}
$$

The constant $z_{s}$ is related to the radial polydispersity, $\sigma_{s}^{2}$ $=1 /\left(z_{s}+1\right)$. One can imagine two approaches with respect to the determination of the value of $z_{s}$; first, $z_{s}$ can be treated as a constant to be fitted to the experimental value, second, one could determine $z_{s}$ from a minimization of the free energy with respect to $z_{s}$, so that $z_{s}$ is expressed in terms of temperature, the total volume and surface area, and the curvature coefficients. The latter approach is more fundamental but mathematically more complex. However, if we assume $\sigma_{s}^{2} \ll 1$ (and later $\sigma_{c}^{2} \ll 1$ ), which is usually a very good approximation, and only keep track of the leading contributions to the free energy, the minimization can be carried out analytically giving explicit expressions for the radial polydispersities.

With the distribution in Eq. (4.5) in terms of $z_{s}$ and the variables $N_{s}$ and $R_{s}$, insertion of $N_{s}(n)$ into the free energy in Eq. (4.1) and integration over $n$ leaves us with the following expression for the free energy of polydisperse spheres:

$$
\frac{F_{s}}{\pi k}=N_{s}\left\{-\frac{16}{R_{0}} R_{s}+4(2+x)+t\left[\ln \left(\frac{\phi r_{0, s}}{R_{s}}\right)+\alpha_{1}\right]\right\},
$$

with the volume and area constraints given by

$$
\begin{aligned}
& A=N_{s} 4 \pi \alpha_{2} R_{s}^{2}, \\
& V_{\text {tot }}=N_{s} \frac{4 \pi}{3} \alpha_{3} R_{s}^{3} .
\end{aligned}
$$

The functions $\alpha_{1}, \alpha_{2}$, and $\alpha_{3}$ appearing in Eqs. (4.7) and (4.8) are defined as

$$
\begin{aligned}
\alpha_{1} & \equiv \ln \left(\frac{z_{s}+1}{\Gamma\left(z_{s}+1\right)}\right)+z_{s} \psi\left(z_{s}+1\right)-z_{s}-2 \\
& \approx-\frac{1}{2} \ln \left(\sigma_{s}^{2}\right)-\frac{3}{2}-\frac{1}{2} \ln (2 \pi)+\frac{1}{3} \sigma_{s}^{2}, \\
\alpha_{2} & \equiv \frac{z_{s}+2}{z_{s}+1} \approx 1+\sigma_{s}^{2}, \\
\alpha_{3} & \equiv \frac{\left(z_{s}+2\right)\left(z_{s}+3\right)}{\left(z_{s}+1\right)^{2}} \approx 1+3 \sigma_{s}^{2},
\end{aligned}
$$

with $\psi(x)$ Euler's psi function. Apart from the presence of the functions $\alpha_{1}, \alpha_{2}$, and $\alpha_{3}$, the expression for the free energy in Eq. (4.7) is the same as the free energy of the monodisperse droplets [Eq. (3.5)].

As in the previous sections, the radius of the spheres is directly determined by the constraints in Eq. (4.8) giving $R_{s}=3 \alpha_{2} / \alpha_{3} \approx 3-6 \sigma_{s}^{2}$ in units of $\omega$. Insertion into the free energy in Eq. (4.7) and differentiation with respect to $\sigma_{s}$ allows the determination of the radial polydispersity. One finds that in an expansion in $\sigma_{s}^{2} \ll 1, \sigma_{s}$ is determined by the following equation, which can readily be solved numerically:

$$
\begin{aligned}
0= & -\frac{t}{24}+\sigma_{s}^{2}\left[-\frac{4}{R_{0}}+2+x\right]+\frac{t}{8} \sigma_{s}^{2} \ln \left(\frac{\phi^{2} r_{0, s}^{2}}{18 \pi \sigma_{s}^{2}}\right) \\
& -\frac{11}{36} t \sigma_{s}^{2}+\frac{t}{12} \sigma_{s}^{4} \ln \left(\frac{\phi^{2} r_{0, s}^{2}}{18 \pi \sigma_{s}^{2}}\right)+\mathcal{O}\left(\sigma_{s}^{4}\right) .
\end{aligned}
$$

Furthermore, the solubilization limit and transition to the lamellar phase are calculated. 


\section{Solubilization limit}

The solubilization limit is given by

$$
\begin{aligned}
\frac{1}{R_{0}}= & \frac{1}{6}(2+x)+\frac{t}{48} \ln \left(\frac{\phi^{2} r_{0, s}^{2}}{18 \pi \sigma_{s}^{2}}\right)-\frac{t}{16} \\
& +\sigma_{s}^{2}\left[\frac{1}{3}(2+x)+\frac{t}{24} \ln \left(\frac{\phi^{2} r_{0, s}^{2}}{18 \pi \sigma_{s}^{2}}\right)-\frac{t}{36}\right]+\mathcal{O}\left(\sigma_{s}^{4}\right),
\end{aligned}
$$

with $\sigma_{s}$ now determined by

$$
\begin{aligned}
0= & -\frac{t}{24}+\frac{1}{3} \sigma_{s}^{2}(2+x)+\frac{t}{24} \sigma_{s}^{2} \ln \left(\frac{\phi^{2} r_{0, s}^{2}}{18 \pi \sigma_{s}^{2}}\right)-\frac{t}{18} \sigma_{s}^{2} \\
& +\frac{t}{12} \sigma_{s}^{4} \ln \left(\frac{\phi^{2} r_{0, s}^{2}}{18 \pi \sigma_{s}^{2}}\right)+\mathcal{O}\left(\sigma_{s}^{4}\right) .
\end{aligned}
$$

\section{Transition to the lamellar phase}

The transition to the lamellar phase is derived by setting $F_{s}=0$ in Eq. (4.7),

$$
\begin{aligned}
\frac{1}{R_{0}}= & \frac{1}{12}(2+x)+\frac{t}{96} \ln \left(\frac{\phi^{2} r_{0, s}^{2}}{18 \pi \sigma_{s}^{2}}\right)-\frac{t}{32} \\
& +\sigma_{s}^{2}\left[\frac{1}{6}(2+x)+\frac{t}{48} \ln \left(\frac{\phi^{2} r_{0, s}^{2}}{18 \pi \sigma_{s}^{2}}\right)-\frac{t}{72}\right]+\mathcal{O}\left(\sigma_{s}^{4}\right),
\end{aligned}
$$

with $\sigma_{s}$ determined by

$$
\begin{aligned}
0= & -\frac{t}{24}+\frac{2}{3} \sigma_{s}^{2}(2+x)+\frac{t}{12} \sigma_{s}^{2} \ln \left(\frac{\phi^{2} r_{0, s}^{2}}{18 \pi \sigma_{s}^{2}}\right)-\frac{13}{72} t \sigma_{s}^{2} \\
& +\frac{t}{12} \sigma_{s}^{4} \ln \left(\frac{\phi^{2} r_{0, s}^{2}}{18 \pi \sigma_{s}^{2}}\right)+\mathcal{O}\left(\sigma_{s}^{4}\right) .
\end{aligned}
$$

\section{B. Spherocylinders}

The inclusion of polydispersity in the distribution of the radius of the cylindrical microemulsion structures follows along the same lines as the spherical droplets. We first consider the free energy of a distribution of cylinders with radius $R_{c}=n r_{0, c}$,

$$
\begin{aligned}
\frac{F_{c}}{\pi k}= & \sum_{n} N_{c}(n)\left\{-\frac{4 L}{R_{0}}+\frac{L}{n r_{0, c}}-\frac{16}{R_{0}} n r_{0, c}+4(2+x)\right. \\
& \left.+t\left[\ln \left(\frac{N_{c}(n) v_{0, c} l_{0}}{V L}\right)-\frac{L}{l_{0}}-1\right]\right\},
\end{aligned}
$$

with the volume and surface area now given by

$$
\begin{aligned}
& A=\sum_{n} N_{c}(n) 2 \pi\left[L n r_{0, c}+2\left(n r_{0, c}\right)^{2}\right], \\
& V_{\mathrm{tot}}=\sum_{n} N_{c}(n) \frac{\pi}{3}\left[3 L\left(n r_{0, c}\right)^{2}+4\left(n r_{0, c}\right)^{3}\right] .
\end{aligned}
$$

The summation is replaced by an integration over $n$ and a Schultz-distribution is assumed for the radial distribution,

$$
N_{c}(n)=\frac{N_{c} r_{0, c}}{R_{c}} \frac{\left(z_{c}+1\right)^{z_{c}+1}}{\Gamma\left(z_{c}+1\right)}\left(\frac{n r_{0, c}}{R_{c}}\right)^{z_{c}} e^{-\left(z_{c}+1\right) n r_{0, c} / R_{c}},
$$

where $N_{c}$ and $R_{c}$ are the total number of cylinders and average radius defined by

$$
\begin{aligned}
& N_{c} \equiv \int_{0}^{\infty} d n N_{c}(n), \\
& R_{c} \equiv \frac{1}{N_{c}} \int_{0}^{\infty} d n N_{c}(n) n r_{0, c} .
\end{aligned}
$$

Again an assumption needs to be made concerning the lengthscales $r_{0, c}, v_{0, c}$, and $l_{0}$. We proceed by assuming that $r_{0, c}$ is a microscopic constant and $v_{0, c}$ and $l_{0}$ to be given by the previous expressions [cf. Eqs. (3.19) and (3.20)],

$$
\begin{aligned}
& v_{0, c} \approx \pi R_{c}^{2} l_{0}, \\
& l_{0} \approx R_{c} .
\end{aligned}
$$

The resulting free energy is then obtained by inserting the distribution in Eq. (4.17) into Eq. (4.15) and carry out the integration,

$$
\begin{aligned}
\frac{F_{c}}{\pi k}= & N_{c}\left\{-\frac{4 L}{R_{0}}+\alpha_{4} \frac{L}{R_{c}}-\frac{16}{R_{0}} R_{c}+4(2+x)\right. \\
& \left.+t\left[\ln \left(\frac{\phi R_{c} r_{0, c}}{L^{2}}\right)-\frac{L}{l_{0}}+\alpha_{5}\right]\right\},
\end{aligned}
$$

with the volume and surface area given by

$$
\begin{aligned}
& A=N_{c} 2 \pi R_{c}\left(L+2 \alpha_{6} R_{c}\right), \\
& V_{\mathrm{tot}}=N_{c} \frac{\pi}{3} R_{c}^{2}\left(3 \alpha_{6} L+4 \alpha_{7} R_{c}\right) .
\end{aligned}
$$

Furthermore we have defined

$$
\begin{aligned}
\alpha_{4} & \equiv \frac{z_{c}+1}{z_{c}}=1+\sigma_{c}^{2}, \\
\alpha_{5} & \equiv \ln \left(\frac{z_{c}+1}{\Gamma\left(z_{c}+1\right)}\right)+z_{c} \psi\left(z_{c}+1\right)-z_{c}-2 \\
& \approx-\frac{1}{2} \ln \left(\sigma_{c}^{2}\right)-\frac{3}{2}-\frac{1}{2} \ln (2 \pi)+\frac{1}{3} \sigma_{c}^{2}, \\
\alpha_{6} & \equiv \frac{z_{c}+2}{z_{c}+1} \approx 1+\sigma_{c}^{2}, \\
\alpha_{7} & \equiv \frac{\left(z_{c}+2\right)\left(z_{c}+3\right)}{\left(z_{c}+1\right)^{2}} \approx 1+3 \sigma_{c}^{2} .
\end{aligned}
$$

In the case that only cylinders are present, the free energy in Eq. (4.20) needs to be minimized with respect to $R_{c}, L, \sigma_{c}$, and $N_{c}$ keeping the volume and surface area constraints in Eq. (4.21) in mind. One finds that $R_{c}, \sigma_{c}$, and $L$ are to leading order in $1 / L$ given by 


$$
\begin{aligned}
R_{c}=2\left(1-2 \sigma_{c}^{2}\right)+\frac{8}{3} \frac{1}{L}+\mathcal{O}\left(\frac{1}{L^{2}}\right), \\
\sigma_{c}^{2}=\frac{1}{L} \frac{t}{3-\frac{8}{R_{0}}-2 t}+\mathcal{O}\left(\frac{1}{L^{2}}\right), \\
t \ln (L)=\frac{4}{9}(7+6 x)-\frac{64}{9} \frac{1}{R_{0}} \\
\quad+\frac{t}{3} \ln \left(\frac{2 \phi^{2} r_{0, c}^{2}}{\pi t}\left(3-\frac{8}{R_{0}}-2 t\right)\right)+\frac{23}{9} t+\mathcal{O}\left(\frac{1}{L}\right) .
\end{aligned}
$$

Furthermore, the solubilization limit and transition to the lamellar phase can be determined.

\section{Solubilization limit}

For a system of only cylinders one finds for the solubilization limit,

$$
\frac{1}{R_{0}}=\frac{1}{4}(1-t)+\frac{1}{12}\left(8-14 t-3 t^{2}\right) \frac{1}{L}+\mathcal{O}\left(\frac{1}{L^{2}}\right),
$$

with $\sigma_{c}^{2}$ and the cylinder length $L$ to leading order given by

$$
\begin{aligned}
& \sigma_{c}^{2}=\frac{t}{L}+\mathcal{O}\left(\frac{1}{L^{2}}\right), \\
& t \ln (L)=\frac{4}{3}(2+x)+\frac{t}{3} \ln \left(\frac{2 \phi^{2} r_{0, c}^{2}}{\pi t}\right)+\frac{13}{3} t+\mathcal{O}\left(\frac{1}{L}\right) .
\end{aligned}
$$

\section{Transition to the lamellar phase}

The transition to the lamellar phase for the cylinders alone is

$$
\frac{1}{R_{0}}=\frac{1}{8}(1-t)-\frac{3}{8} t \frac{1}{L}+\mathcal{O}\left(\frac{1}{L^{2}}\right),
$$

with $\sigma_{c}^{2}$ and the cylinder length $L$ to leading order given by

$$
\begin{aligned}
\sigma_{c}^{2}=\frac{t}{2-t} & \frac{1}{L}+\mathcal{O}\left(\frac{1}{L^{2}}\right) \\
t \ln (L)= & \frac{4}{9}(1+6 x)+\frac{t}{3} \ln \left(\frac{2 \phi^{2} r_{0, c}^{2}}{\pi t}(2-t)\right)+\frac{31}{9} t \\
& +\mathcal{O}\left(\frac{1}{L}\right) .
\end{aligned}
$$

\section{Spheres and spherocylinders}

Finally, we introduce the volume fractions $v_{s}$ and $v_{c}$ and obtain the free energy of the system containing both spheres and cylinders using Eqs. (4.7) and (4.20),

$$
\begin{aligned}
\frac{\omega^{3} F}{k V_{\mathrm{tot}}}= & \frac{v_{s}}{\alpha_{3}}\left\{-\frac{12}{R_{0}} \frac{1}{R_{s}^{2}}+3(2+x) \frac{1}{R_{s}^{3}}+t \frac{3}{4 R_{s}^{3}}\right. \\
& \left.\times\left[\ln \left(\frac{\phi v_{s} r_{0, s}}{R_{s}}\right)+\alpha_{1}\right]\right\}+\frac{3 v_{c} L}{\left(3 \alpha_{6} L+4 \alpha_{7} R_{c}\right)} \\
& \times\left\{-\frac{4}{R_{0}} \frac{1}{R_{c}^{2}}+\frac{\alpha_{4}}{R_{c}^{3}}-\frac{16}{R_{0}} \frac{1}{R_{c} L}+4(2+x) \frac{1}{R_{c}^{2} L}\right. \\
& \left.+t \frac{1}{R_{c}^{2} L}\left[\ln \left(\frac{\phi v_{c} R_{c} r_{0, c}}{L^{2}}\right)-\frac{L}{l_{0}}+\alpha_{5}\right]\right\},
\end{aligned}
$$

with the volume and area constraints

$$
\begin{aligned}
& v_{s}+v_{c}=1, \\
& \frac{3 \alpha_{2}}{\alpha_{3}} \frac{v_{s}}{R_{s}}+6 \frac{v_{c}}{R_{c}} \frac{\left(L+2 \alpha_{2} R_{c}\right)}{\left(3 \alpha_{2} L+4 \alpha_{3} R_{c}\right)}=1 .
\end{aligned}
$$

Besides temperature $t$ and the curvature coefficients $x$ and $1 / R_{0}$, the inclusion of radial polydispersity has left us with the additional parameters $r_{0, s}$ and $r_{0, c}$. Furthermore, the volume and area constraints manifest themselves in the presence of the total volume fraction $\phi$ and length scale $\omega$.

The free energy in Eq. (4.28) is expressed in terms of the seven variables $R_{s}, R_{c}, L, z_{s}, z_{c}, v_{s}$, and $v_{c}$ to be determined by minimization of the free energy with the constraints in Eq. (4.29). This has been done, numerically, with the result shown in Fig. 3. In this example we have set $r_{0, s}$ $=1$ and $r_{0, c}=1$ (in units of $\omega$ ). Similar results are obtained when different values for $r_{0, s}$ and $r_{0, c}$ are assumed. In general, lowering $r_{0}$ shifts the phase boundaries uniformly to the right in the phase diagrams depicted in Fig. $3(\Delta x \approx 0.17$ per factor 10 in $r_{0}$ ).

The general shape of the phase diagram and the evolution of the cylinder volume fraction [Fig. 3(a)] and cylinder length [Fig. 3(b)] is the same as in Fig. 2. The advantage of including radial polydispersity therefore mainly lies in the fact that explicit values for the spherical [Fig. 3(c)] and cylindrical [Fig. 3(d)] radial polydispersities can be provided. It is concluded that $\sigma_{s}$ and $\sigma_{c}$ decrease going in the direction of the lamellar phase. Furthermore, the radial polydispersities also decrease in the direction of increasing $x \equiv \bar{k} / k$ with the important distinction, however, that $\sigma_{s}$ takes on a minimum polydispersity of about $14 \%$ (for the few spherical droplets that remain in this region) while $\sigma_{c}$ vanishes $[\propto 1 / L$; see Eq. (4.23)]. An interesting experimental consequence of the results in Figs. 3(c) and 3(d) is that in the case that the inversion temperature is approached from below $(T<\bar{T})$, the radial polydispersity decreases with increasing temperature. This decrease in polydispersity with increasing temperature is then purely a result from the intricate interplay between the constraints, entropy, and curvature energy.

We now show how the results of this section can be compared to experimental phase diagrams. To make this comparison more transparent it should be reminded that we can take $k$ and $\bar{k}$ as approximately constant (fixed $x$ and $t$ ) and treat $1 / R_{0}$ as the "temperature variable" $1 / R_{0} \propto(T-\bar{T})$. In Fig. 4(a) the experimental microemulsion phase diagram is shown as a function of the oil to water ratio $\alpha \equiv o /(w$ 

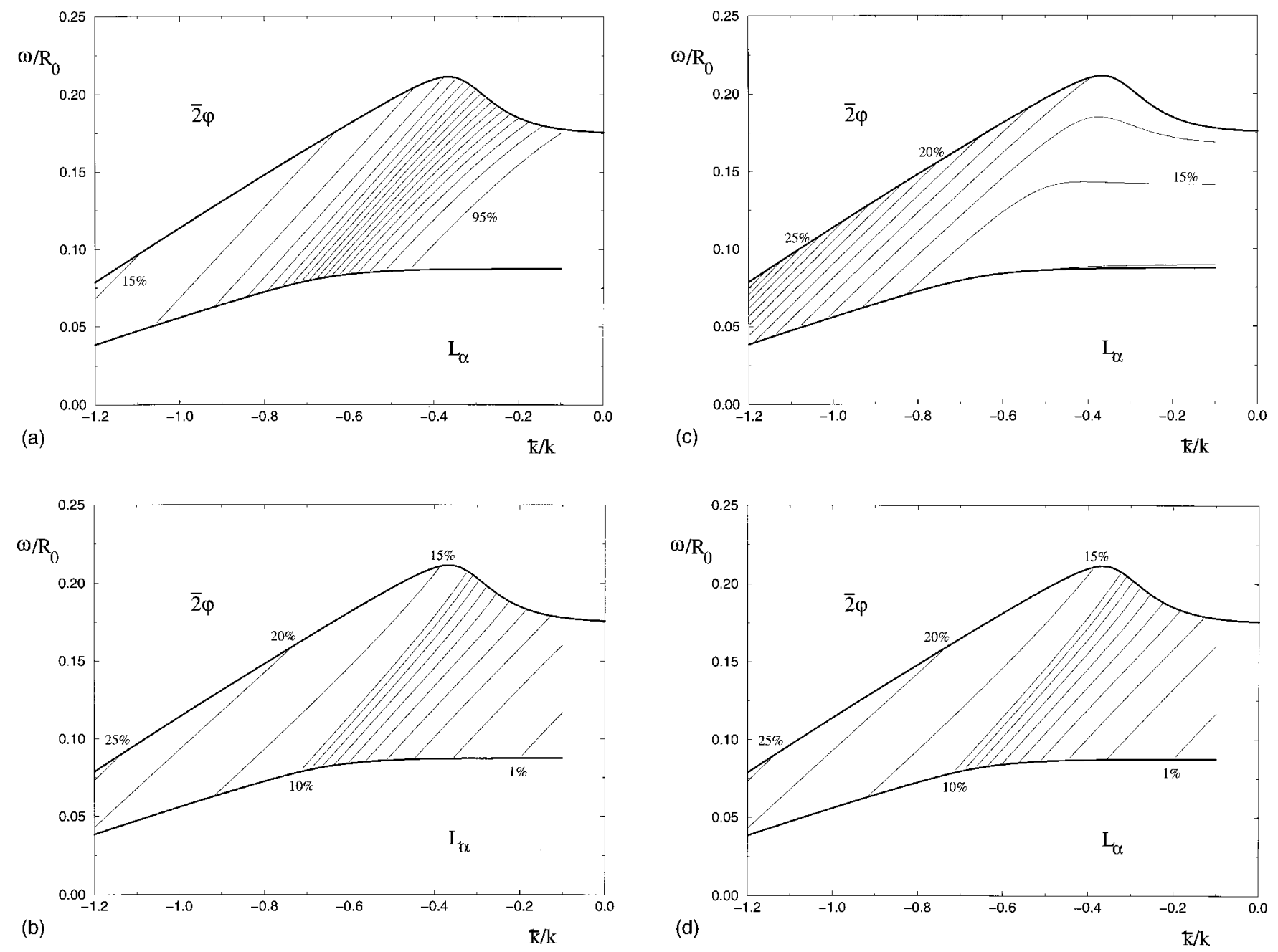

FIG. 3. Microemulsion phase diagram with translational entropy, cylinder length polydispersity, and radial polydispersities, as a function of $\omega / R_{0}$ and $\bar{k} / k$. We have chosen $\phi=0.05, t=0.3$ and $r_{0, s}=r_{0, c}=1$ (in units of $\omega$ ). (a) shows the cylinder volume fraction, $v_{c}=15 \%-95 \%$ in steps of 5\%; (b) shows the average cylinder length, $L=3-3000$ (in units of $\omega$ ) in logarithmic steps; (c) shows the radial polydispersity of the sphere, $\sigma_{s}=14 \%-27 \%$ in steps of $1 \%$; (d) shows the radial polydispersity of the cylinder, $\sigma_{c}=1 \%-10 \%$ in steps of $1 \%$ and $\sigma_{c}=10 \%-25 \%$ in steps of $5 \%$.

$+o$ ) and temperature $T$ at constant surfactant concentration $\gamma \equiv s /(w+s+o)$, with $w, s$, and $o$ the water, surfactant, and oil weight fraction, respectively. The usual structural evolution within the one-phase region, bounded by lamellar phases $\left(L_{\alpha}\right)$ and two two-phase regions $(\overline{2}$ and 2$)$, is sketched. The evolution shown is from spherical water droplets in oil (lower right corner) via a bicontinuous phase to oil droplets in water (upper right corner). ${ }^{31}$ The region where the results of this section are expected to be most applicable is the region close to the droplet region, not too close to the lamellar region and not too close to the bicontinuous region. We have calculated the evolution of the cylinder volume fraction taking $t=0.3, r_{0, s}=r_{0, c}=1$ (in units of $\omega$ ) and $\gamma=0.2$ in Fig. 4(b) which roughly corresponds to the region enclosed by the dashed line in Fig. 4(a). In comparison with the usual sketch of the structural evolution, which has emerged on the basis of extensive experimental effort, ${ }^{31}$ it is noted that an increase in the number of cylinders (as well as average length) more prominantly occurs in the direction of increasing temperature $\left(1 / R_{0} \rightarrow 0\right)$ than with decreasing $\alpha$.

In Fig. 5(a), the microemulsion phase diagram is shown as a function of surfactant concentration $\gamma$ and temperature $T$ at constant oil to water ratio $\alpha=0.5$. This is the Kahlweit "fish"-diagram ${ }^{2}$ showing a three-phase region (3) and two two-phase regions ( $\overline{2}$ and 2$)$. Again, the evolution is sketched from spherical water droplets in oil via a bicontinuous phase to oil droplets in water. ${ }^{32}$ The evolution of the cylinder volume fraction, taking $t=0.3$ and $r_{0, s}=r_{0, c}=1$ (in units of $\omega$ ), is shown in Fig. 5(b) which roughly corresponds to the region enclosed by the dashed line in Fig. 5(a). It should be noted, however, that $\alpha=0.5$ corresponds to a rather substantial droplet volume fraction $\phi$ violating the assumption $\phi \ll 1$, so that the comparison between Figs. 5(a) and 5(b) should be taken only as a qualitative comparison.

\section{SUMMARY AND DISCUSSION}

We have showed that the Helfrich free energy model can be used to describe the sphere to cylinder transition in a one-phase region microemulsion system. In order for our description to be as realistic as possible we have included, be- 

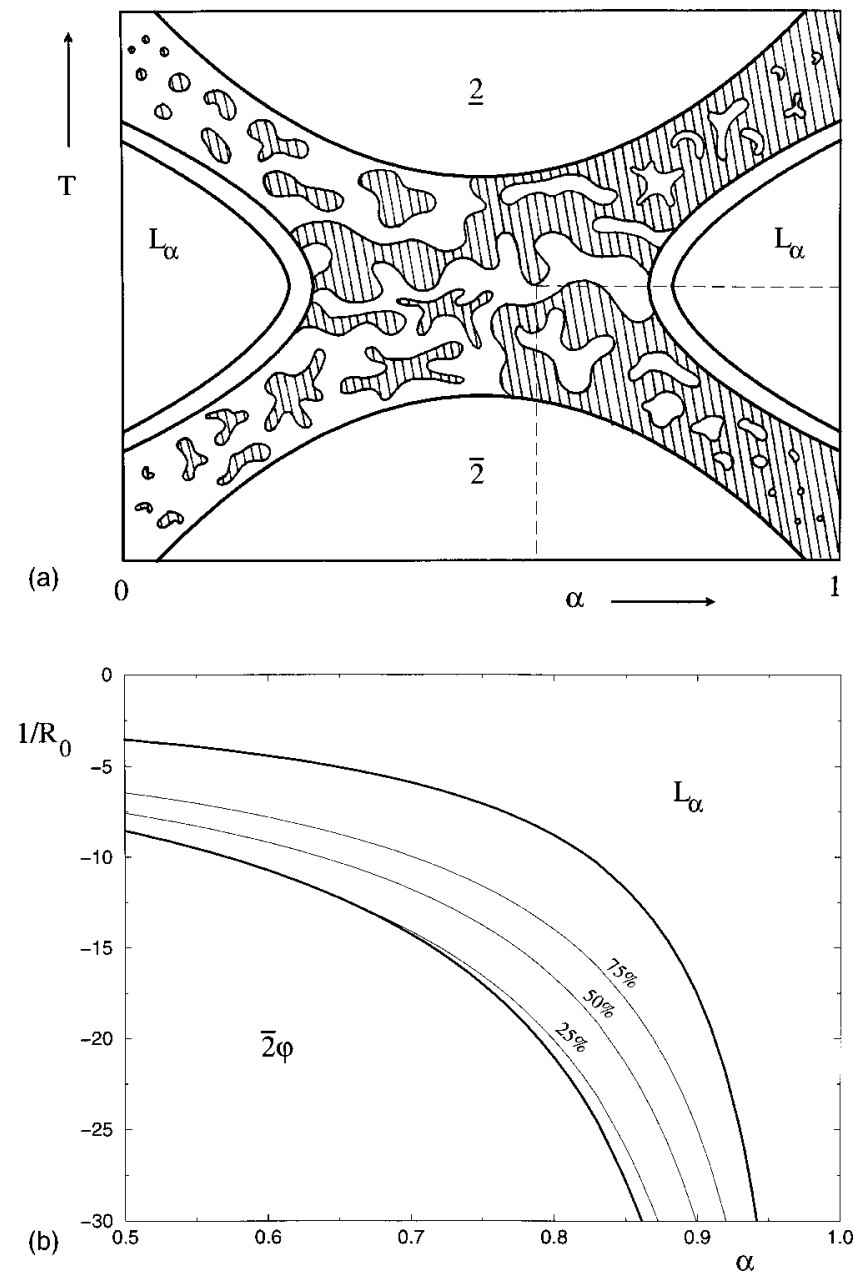

FIG. 4. Microemulsion phase diagram as a function of the oil to water ratio $\alpha$ and (a) temperature or (b) $1 / R_{0}$, at fixed surfactant concentration $\gamma$. In (a) the usual structural evolution within the one-phase region is sketched. The one-phase region is bounded by two lamellar phase regions $\left(L_{\alpha}\right)$ and two two-phase regions ( $\overline{2}$ and 2$)$. The region enclosed by the dashed lines shows the region where the theory is expected to be most applicable. This region is shown in (b) which was calculated taking $t=0.3, r_{0, s}=r_{0, c}=1$ (in units of $\omega)$ and $\gamma=0.2$. Also shown is the cylinder volume fraction, $v_{c}$ $=25 \%-75 \%$ in steps of $25 \%$.

sides translation entropy, cylinder length polydispersity, and radial polydispersity. The model presented here has a number of limitations that should be discussed.

(i) We have neglected all interactions between the different structures present. This effectively means that the validity of our analysis is limited to small volume fractions, $\phi \ll 1$. For a realistic description at higher volume fractions, the sphere-sphere, sphere-cylinder, and cylinder-cylinder interaction energy needs to be included. Treating the spherical droplets as hard spheres or sticky hard spheres, ${ }^{8}$ which may or may not be a valid assumption, ${ }^{33}$ the sphere-sphere interaction energy can be well described by a Percus-Yevick approximation, but little is known about the other interactions thus seriously hampering the extension to higher volume fractions.

(ii) Another drawback of the model is the neglect of the possible existence of other phases. In the direction of zero spontaneous curvature $\left(1 / R_{0} \rightarrow 0\right)$ it is expected that the cylinders formed start to branch ${ }^{17}$ when they increase in length
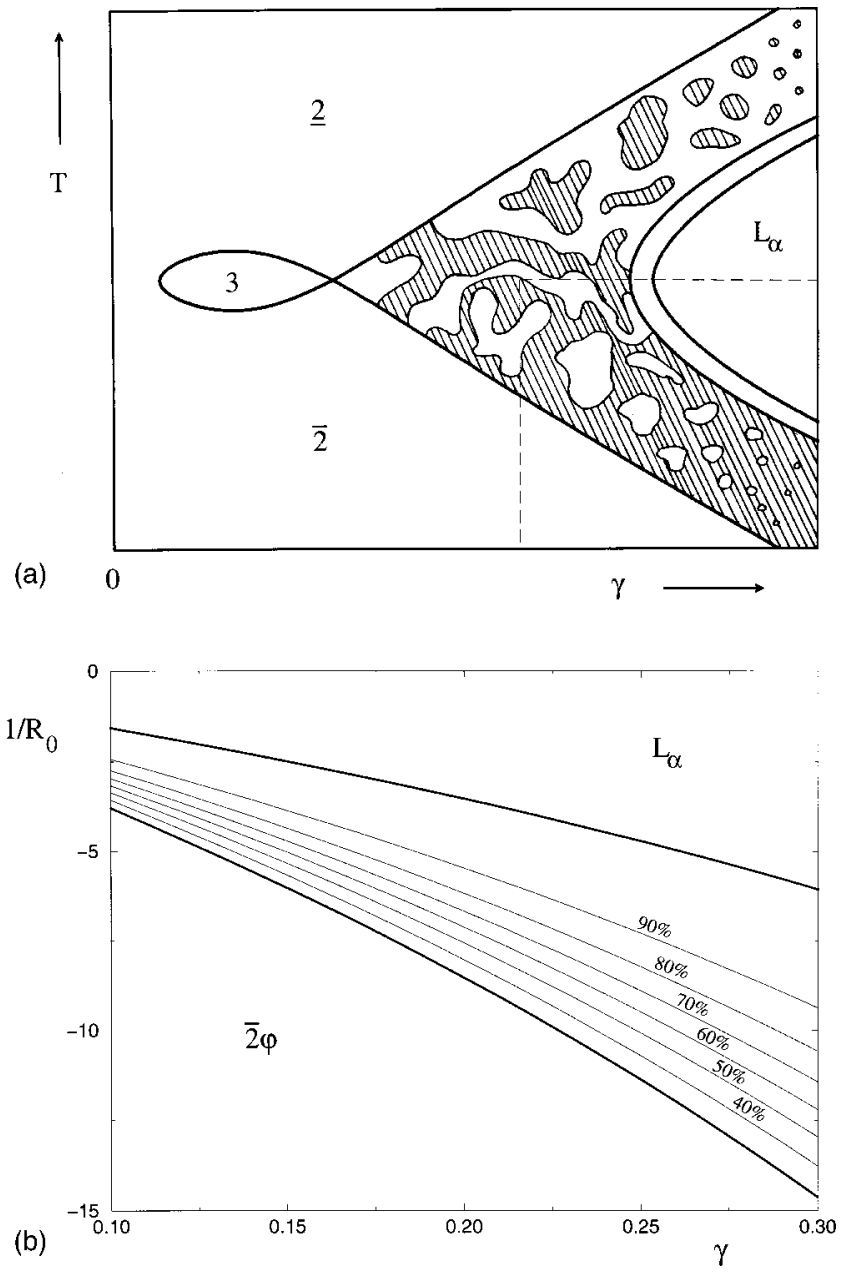

FIG. 5. Microemulsion phase diagram as a function of surfactant concentration $\gamma$ and (a) temperature or (b) $1 / R_{0}$, at fixed oil to water ratio, $\alpha$ $=0.5$. In (a) the usual structural evolution within the one-phase region is sketched. This is the "fish"-diagram showing a three-phase region (3) and two two-phase regions $(\overline{2}$ and 2$)$. The region enclosed by the dashed lines shows the region where the theory is expected to be most applicable. This region is shown in (b) which was calculated taking $t=0.3$ and $r_{0, s}=r_{0, c}$ $=1$ (in units of $\omega$ ). Also shown is the cylinder volume fraction, $v_{c}$ $=40 \%-90 \%$ in steps of $10 \%$.

and ultimately form a bicontinuous phase that competes with the formation of a lamellar structure. At present we have limited ourselves to the calculation of the point where the free energy changes sign, and the lamellar phase is formed, hereby neglecting the narrow region of microemulsionlamellar phase coexistence or entropy considerations for the lamellar phase. It should therefore be concluded that our theoretical analysis is most valid close to the solubilization limit describing the onset of the sphere to cylinder transition.

(iii) The theory presented here has a mean-field character. This means that even though certain fluctuations around the mean are taken into consideration-for example those fluctuations that only change the radius-shape fluctuations are not taken into account. The result is that our calculated values for the radial polydispersity are a lower limit to the experimental value. Especially in the case of the radial polydispersity of very long cylinders, the contribution to the radial polydispersity of a uniform fluctuation changing only the radius of the cylinder becomes negligible compared to 
the contribution from undulatory shape fluctuations.

(iv) Many assumptions and approximations have been made with regard to the consideration of entropy-effects. These include the Flory-Huggins approximation for the cylinder entropy and the assumptions made on the magnitude of the "entropy length scales" $v_{0, s}, v_{0, c}, l_{0}, r_{0, s}$, and $r_{0, c}$. Although we have not shown it in great detail, it turns out that details concerning the choice of the entropy terms only deform the phase diagram slightly without changing the overall character of it. The same holds for the influence on the calculation of the structural parameters, $v_{c}, R_{s}, R_{c}, \sigma_{s}$, and $\sigma_{c}$. Only the average cylinder length $L$ turns out to be logarithmically sensitive to the details of the model and the numbers presented here should therefore only be taken as an indication.

\section{ACKNOWLEDGMENTS}

We would like to thank M. Borkovec, J. C. Eriksson, and U. Heissner for interesting discussions. The work of W.F.C.S. has been supported by the Netherlands Foundation for Chemical Research (CW) in collaboration with the Netherlands Technology Foundation (STW).

${ }^{1}$ D. F. Evans and H. Wennerström, The Colloidal Domain, 2nd ed. (Wiley, New York, 1999); H. T. Davis, J. F. Bodet, L. E. Scriven, and W. G. Miller, in Physics of Amphiphilic Layers, edited by J. Meunier, D. Langevin, and N. Bocarra (Springer, Berlin, 1987); Y. Chevalier and T. N. Zemb, Rep. Prog. Phys. 53, 279 (1990); J. Eastoe, B. H. Robinson, D. C. Steytler, and D. Thorn-Leeson, Adv. Colloid Interface Sci. 36, 1 (1991).

${ }^{2}$ M. Kahlweit, R. Strey, and G. Busse, J. Phys. Chem. 94, 3881 (1990).

${ }^{3}$ D. Chatenay, W. Urbach, A. M. Cazabat, and D. Langevin, Phys. Rev. Lett. 54, 2253 (1985); S.-H. Chen, S.-L. Chang, and R. Strey, J. Chem. Phys. 93, 1907 (1990); R. Johannson, M. Almgren, and J. Alsins, J. Phys. Chem. 95, 3819 (1991); P. D. I. Fletcher and J. S. Morris, Colloids Surf., A 98, 147 (1995); G. J. M. Koper, W. F. C. Sager, J. Smeets, and D. Bedeaux, J. Phys. Chem. 99, 13291 (1995).

${ }^{4}$ S. Bhattacharya, J. P. Stokes, M. W. Kim, and J. S. Huang, Phys. Rev. Lett. 55, 1884 (1985); C. Cametti, P. Codatefano, P. Tartagla, S.-H. Chen, and J. Rouch, Phys. Rev. A 45, R5358 (1992); W. Sager, W. Sun, and H.-F. Eicke, Prog. Colloid Polym. Sci. 89, 282 (1992); L. Schlicht, J. H. Spilgies, F. Runge, S. Lipgens, S. Boye, D. Schübel, and G. Ilgenfritz, Biophys. Chem. 58, 39 (1996); L. M. M. Nazario, T. A. Hatton, and J. P. S. G. Crespo, Langmuir 12, 6326 (1996).

${ }^{5}$ J. N. Israelachvili, D. J. Mitchell, and B. W. Ninham, J. Chem. Soc., Faraday Trans. 2 72, 1525 (1976).

${ }^{6}$ M. E. Cates and S. J. Candau, J. Phys.: Condens. Matter 2, 6869 (1990).

${ }^{7}$ J. S. Huang and M. W. Kim, Phys. Rev. Lett. 47, 1462 (1981); B. H. Robinson, C. Toprakcioglu, J. C. Dore, and P. Chieux, J. Chem. Soc., Faraday Trans. 1 80, 13 (1984); M. Kotlarchyk, R. B. Stephens, and J. S. Huang, J. Phys. Chem. 92, 1533 (1988); R. Hilfiker, H.-F. Eicke, W. Sager, C. Steeb, U. Hofmeier, and R. Gehrke, Ber. Bunsenges. Phys. Chem. 94, 677 (1990); M. Kotlarchyk, J. S. Huang, and S.-H. Chen, J. Phys. Chem. 89, 4382 (1985); M. Kotlarchyk, S.-H. Chen, and J. S. Huang, ibid. 86, 3273 (1982).
${ }^{8}$ S.-H. Chen, C. Y. Ku, J. Rouch, P. Tartiaglia, C. Cametti, and J. Samseth, J. Phys. IV 3, 143 (1993).

${ }^{9}$ S. Lipgens, D. Schübel, L. Schlicht, J. H. Spilgies, G. Ilgenfritz, J. Eastoe, and R. K. Heenan, Langmuir 14, 1041 (1998).

${ }^{10}$ O. Glatter, R. Strey, K-V. Schubert, and E. W. Kaler, Ber. Bunsenges. Phys. Chem. 100, 323 (1996); J. Brunner-Popela, R. Mittelbach, R. Strey, K-V. Schubert, E. W. Kaler, and O. Glatter, J. Chem. Phys. 110, 10623 (1999).

${ }^{11}$ D. I. Svergun, P. V. Konarev, V. V. Volkov, M. H. J. Koch, W. F. C. Sager, J. Smeets, and E. M. Blokhuis, J. Chem. Phys. 113, 1651 (2000).

${ }^{12}$ W. Helfrich, Z. Naturforsch. C 28, 693 (1973).

${ }^{13}$ For reviews, see Micelles, Membranes, Microemulsions, and Monolayers, edited by W. M. Gelbart, A. Ben-Shaul, and D. Roux (Springer, New York, 1994); Statistical Mechanics of Membranes and Surfaces, edited by D. Nelson, T. Piran, and S. Weinberg (World Scientific, Singapore, 1988); M. Wortis, U. Seifert, K. Berndl, B. Fourcade, M. Rao, and R. Zia, in Dynamical Phenomena at Interfaces, Surfaces and Membranes, edited by D. Beysens, N. Boccara, and G. Forgacs (Nova Science, New York, 1993).

${ }^{14}$ S. A. Safran, Statistical Thermodynamics of Surfaces, Interfaces, and Membranes (Addison-Wesley, Reading, 1994), and references therein.

${ }^{15}$ S. A. Safran, L. A. Turkevich, and P. Pincus, J. Phys. (France) Lett. 45, L-69 (1984).

${ }^{16}$ R. Menes, S. A. Safran, and R. Strey, Phys. Rev. Lett. 74, 3399 (1995).

${ }^{17}$ T. Tlusty and S. A. Safran, J. Phys.: Condens. Matter 12, A-253 (2000); T. Tlusty, S. A. Safran, and R. Strey, Phys. Rev. Lett. 84, 1244 (2000).

${ }^{18}$ M. Borkovec and H.-F. Eicke, Chem. Phys. Lett. 157, 457 (1989); J. S. Huang, S. T. Milner, B. Farargo, and D. Richter, Phys. Rev. Lett. 59, 2600 (1987); B. P. Binks, J. Meunier, O. Abillon, and D. Langevin, Langmuir 5, 415 (1989); H. Kellay, B. P. Binks, and J. Meunier, Phys. Rev. Lett. 70, 1485 (1993)

${ }^{19}$ R. Strey, Colloid Polym. Sci. 272, 1005 (1994).

${ }^{20}$ For a recent review, see D. C. Morse, Curr. Opin. Colloid Interface Sci. 2, 365 (1997).

${ }^{21}$ M. Gradzielski, D. Langevin, and B. Farago, Phys. Rev. E 53, 3900 (1996).

${ }^{22}$ P. J. Flory, Principles of Polymer Chemistry (Cornell University Press, Ithaca, 1981).

${ }^{23}$ K. M. Palmer and D. C. Morse, J. Chem. Phys. 105, 11147 (1996).

${ }^{24}$ P. Sollich, P. B. Warren, and M. E. Cates, "Moment free energies for polydisperse systems" (preprint).

${ }^{25}$ J. Th. G. Overbeek, G. J. Verhoeckx, P. L. de Bruyn, and H. N. W. Lekkerkerker, J. Colloid Interface Sci. 119, 422 (1987); J. Th. G. Overbeek, Prog. Colloid Polym. Sci. 83, 1 (1990).

${ }^{26}$ H. Reiss, J. Colloid Interface Sci. 53, 61 (1975); Adv. Colloid Interface Sci. 7, 1 (1977).

${ }^{27}$ J. C. Eriksson and S. Ljunggren, Prog. Colloid Polym. Sci. 81, 41 (1990).

${ }^{28}$ M. Borkovec, Adv. Colloid Interface Sci. 37, 195 (1992).

${ }^{29}$ The distribution in Eq. (4.3) was investigated further taking dropletdroplet interactions into account via the Percus-Yevick approximation, in C. Guerra, A. M. Somoza, and M. M. Telo da Gama, J. Chem. Phys. 111, 7646 (1999).

${ }^{30}$ G. V. Schultz, J. Phys. Chem. 30, 379 (1935).

${ }^{31} \mathrm{U}$. Olsson and H. Wennerström, Adv. Colloid Interface Sci. 49, 113 (1994).

${ }^{32}$ W. K. Kegel, Ph.D. thesis, University of Utrecht, 1993.

${ }^{33}$ E. M. Blokhuis and W. F. C. Sager, J. Chem. Phys. 110, 3148 (1999); 111, 7062 (1999). 